Modern Physics Letters A

Vol. 24, No. 34 (2009) 2769

World Scientific Publishing Company

\title{
On the definition of matter collineations
}

\author{
Asghar Qadir $^{a}$ and K. Saifullah ${ }^{b}$ \\ ${ }^{a}$ Centre for Advanced Mathematics and Physics \\ National University of Sciences and Technology, Rawalpindi, Pakistan \\ ${ }^{b}$ Department of Mathematics, Quaid-i-Azam University, Islamabad, Pakistan \\ (Electronic address: aqadirmath@yahoo.com,saifullah@qau.edu.pk)
}

\begin{abstract}
It is shown that when the stress-energy tensor of a spacetime is diagonal and is written in the mixed form, its collineations admit infinite dimensional Lie algebras except possibly in the case when the tensor depends on all the spacetime coordinates. The result can be extended for more general second rank tensors.
\end{abstract}


Symmetries of the spacetime metric, $\mathbf{g}$, are given by the Killing equations

$$
£_{\xi} \mathbf{g}=0
$$

where $£_{\xi}$ represents the Lie derivative and $\underline{\xi}$ are called Killing vectors (KVs). If $\mathbf{g}$ in the above equation is replaced by some other tensor the symmetry vectors are called collineations of that tensor[1, 2, 3, 4]. Thus, for example, we define Ricci collineations (RCs) by

$$
£_{\xi} \mathbf{R}=0
$$

When we write the Ricci tensor, $\mathbf{R}$, in covariant form, the above equation can be written in components as

$$
\xi^{c} R_{a b, c}+R_{a c} \xi_{, b}^{c}+R_{b c} \xi_{, a}^{c}=0
$$

If $\mathbf{R}$ in the above equation is replaced by $\mathbf{T}$, the stress-energy tensor, $\underline{\xi}$ is called a matter collineation (MC) $[5,6]$. Similarly we define Weyl and curvature collineations. Applying these symmetry constraints is one way to obtain solutions of the system of Einstein field equations (EFEs)

$$
R_{\mu \nu}-\frac{1}{2} R g_{\mu \nu}=\kappa T_{\mu \nu}
$$

which is highly non-linear and is very difficult to solve otherwise. Ricci colli- neations (RCs), for example, have been used to solve EFEs[7]. These symmetries lead to conservation laws also. Davis et al. $[8,9]$ did the pioneering work on the important role of RCs and the related conservation laws that are admitted by particular types of matter fields. They showed that the existence of isometries and collineations leads to conservation laws in the form of integrals of a dynamical system. They also considered the application of these results to relativistic hydrodynamics and plasma physics. Oliver and Davis[10] obtained conservation expressions for perfect fluids using RCs. The properties of fluid spacetimes admitting RCs were studied by Tsamparlis and Mason[11]. They have studied perfect fluid spacetimes in detail and have also considered a variety of imperfect fluids with cosmological constant and with anisotropic pressure. Apart from these physical considerations symmetries provide an invariant basis for classification of spacetimes also (see, for example, Refs. [7], [12]-[20]).

To actually compute the collineations one needs to write the equations for them in component form ([7], [12]-[20]). Thus the valence[21] of the tensor is relevant. 
In fact, the symmetry algebra is different for the different forms, in general. While it is "natural" to use the covariant forms for the metric and the Ricci tensor, $\mathbf{R}$, for the stress-energy tensor, $\mathbf{T}$, it is far from obvious which form should be used. However, MCs generally discussed in the literature[14, 15, 19, 20] are obtained from the covariant form. The question as to whether one should use the contravariant $\left(T^{\mu \nu}\right)$, covariant $\left(T_{\mu \nu}\right)$ or mixed $\left(T_{\nu}^{\mu}\right)$ form of the stress-energy tensor, becomes more important when one notes that all these forms have their own separate significance in general relativity. While the original field theoretic definition of the tensor comes from $[22]$

$$
T_{\nu}^{\mu}=q_{, \nu}^{\alpha} \frac{\delta L}{\delta q_{, \mu}^{\alpha}}-\delta_{\nu}^{\mu} L,
$$

where $L$ is the Lagrangian density and $q^{\alpha}$ are the generalized coordinates, it is the covariant form that arises naturally in the EFEs. On the other hand, the contravariant form is needed for the Hamiltonian formulation[23]. This ambiguity in the definition of MCs has also been pointed out in Ref. [6].

There are two questions to be considered: (a) which (if any) of the forms can be regarded as "correct"; (b) what can be obtained from each of them? We consider the form obtained from the field theoretic definition and explore question (b). In particular, we show that for a diagonal mixed form stress-energy tensor the Lie algebra for the MC vectors will be infinite dimensional except when it depends on all four spacetime variables in which case it can be finite dimensional. When we say that $T_{\nu}^{\mu}$ is diagonal we mean that it is of the diagonal Segre type[4] and it is diagonal when expressed as a matrix in coordinate components. Further, the MCs are assumed to be smooth, so that they form a Lie algebra. The MC equations in component form are

$$
\xi^{\rho} T_{\nu, \rho}^{\mu}+T_{\rho}^{\mu} \xi_{, \nu}^{\rho}-T_{\nu}^{\rho} \xi_{, \rho}^{\mu}=0
$$

Here the components of $T_{\nu}^{\mu}$ and the MC vector, $\xi^{\mu}$, are functions of the coordinates $x^{\mu}$. We note that while in the covariant (or contravariant) form[14, 15, 19, 20] there are ten equations in four dimensions, in the mixed form we have sixteen equations. It is worth mentioning here that if $T_{\nu}^{\mu}$ is a tensor (as will be discussed later) then these sixteen equations are independent, but if it represents the stress-energy tensor then they are restricted by $T_{\nu}^{\mu} g_{\mu \rho}=T_{\rho}^{\mu} g_{\mu \nu}$. Dropping the summation convention here and hereafter, for the diagonal stress-energy tensor equation (6) can be written as 


$$
\xi_{, \nu}^{\mu}\left(T_{\mu}^{\mu}-T_{\nu}^{\nu}\right)=0, \quad(\mu, \nu=0,1,2,3)
$$

which is a set of 12 equations, for $\mu \neq \nu$, and

$$
\sum_{\rho=0}^{3} \xi^{\rho} T_{\mu, \rho}^{\mu}=0
$$

is a set of 4 equations, as it applies for each $\mu$ separately.

Note that in the first set of twelve equations if $T_{\mu}^{\mu} \neq T_{\nu}^{\nu}(\mu \neq \nu)$ then

$$
\xi_{, \nu}^{\mu}=0
$$

that is, if all the components of the stress-energy tensor are different then for each $\mu, \xi^{\mu}$ can only be a function of $x^{\mu}$. For the next set of four equations (8) we do the analysis by viewing $T_{\mu, \nu}^{\mu}$ as the matrix $\mathbf{A}$ with elements $A_{\nu}^{\mu}$. Now, if $\operatorname{rank}(\mathbf{A})=4$, then $\xi^{\mu}=0$, i.e. the columns of $\mathbf{A}$ are 4 linearly independent vectors in a 4 dimensional space and $\underline{\xi}$ is orthogonal to all of them, and hence, it is a zero vector. Now, if $\operatorname{rank}(\mathbf{A})$ is less than 4 , then $\underline{\xi}$ is an eigenvector of $\mathbf{A}$ with zero eigenvalue and the subspace spanned by these eigenvectors satisfy equations (8). Thus, for example, if $\operatorname{rank}(\mathbf{A})$ is 3 , then there will be $4-3=1$ such eigenvectors and we can say that the vector has one degree of freedom, or the MC algebra can be written[21] as $\infty^{1}$. Note that if $\xi^{a}$ is a solution of equations (8) then for some function $f, f \xi^{a}$ is also a solution but this will be subject to equations (7) which may only increase the degree of freedom. Further, if $T_{\nu}^{\mu}$ depends on one variable only then the rank of $\mathbf{A}$ is 1 at most and degrees of freedom will at least be $\infty^{3}$. If it depends on two variables the maximum rank will be 2 and degrees of freedom will at least be $\infty^{2}$; if three variables the maximum rank is 3 . Similarly, if the rank is 0 (for example, when the tensor is constant) it is $\infty^{4}$.

In the above we assumed that all the components of $T_{\mu}^{\mu}$ are different. If we drop this assumption we see that, when equations (7) are imposed on the above results different possibilities arise, but in all these cases the degree of freedom will only increase.

In order to show that a finite dimensional algebra is indeed admitted, we construct an example. For non-trivial collineation vector with finite dimensional Lie algebras to exist it is necessary that the rank of the matrix $\left(T_{\mu, \nu}^{\mu}\right)$ be 3 . For example, take

$$
T_{0}^{0}=t+x+y, \quad T_{1}^{1}=t+x+z,
$$




$$
T_{2}^{2}=t+y+z, \quad T_{3}^{3}=x+y-2 z,
$$

where $x^{0}=t, x^{1}=x, x^{2}=y, x^{3}=z$ are arbitrary coordinates and not necessarily Cartesian coordinates. The solution is

$$
\xi=-2 \frac{\partial}{\partial t}+\frac{\partial}{\partial x}+\frac{\partial}{\partial y}+\frac{\partial}{\partial z},
$$

which is non-trivial. Note that for simplicity we have assumed units in which the tensor can be given in the same units as the coordinates. More generally, appropriate constants could have been inserted.

Thus we conclude that for a diagonal stress-energy tensor written in the mixed form, the MCs (defined by equations (6)) admit an infinite dimensional Lie algebra except possibly when the tensor depends on all the spacetime coordinates. If rank $(\mathbf{A})$ is zero, the minimum degrees of freedom are 4; if one, the minimum is 3; two, 2; three, 1. If $\operatorname{rank}(\mathbf{A})=4$ the $M C$ vector is zero.

Let us assume that for a class of spacetimes there exists a coordinate system in which one or more of the coordinates are missing from all the components of the tensor. Then the algebra will become infinite dimensional. Thus in the case of static spacetimes, for example, the Lie algebras for diagonal mixed $\mathrm{MC}$ vectors are infinite dimensional. For example Refs. $[14,15,19,20]$ dealing with static spacetimes use the covariant form, $T_{\mu \nu}$, and obtain some finite dimensional Lie algebras. Here we see that they are all infinite dimensional Lie algebra for the mixed form $T_{\nu}^{\mu}$.

It is worth mentioning here that while we considered the stress-energy tensor, the results hold for any tensor of rank two in a space of any dimensions. Thus we can state the following result.

For a diagonal tensor of rank two written in the mixed form, the Lie symmetries (as defined by equations (6)) have infinite dimensional Lie algebras except possibly when the tensor depends on all the coordinate variables. The maximum number of degrees of freedom is $n^{2}$ and the minimum zero, where $n$ is the dimension of the space.

For example, consider the Friedmann-Robertson-Walker spacetime in stereographic coordinates (for $k=0$, i.e. the flat case)

$$
d s^{2}=d \tilde{t}^{2}-S(\tilde{t})^{2}\left(d x^{2}+d y^{2}+d z^{2}\right),
$$

where $S$ is a non-zero function of $\tilde{t}$. Its stress-energy tensor is

$$
\begin{aligned}
& T_{0}^{0}=3 \dot{S}^{2} / S^{2}=A(\tilde{t}) \\
& T_{1}^{1}=T_{2}^{2}=T_{3}^{3}=-\left(\dot{S}^{2}+2 S \ddot{S}\right)=B(\tilde{t}) .
\end{aligned}
$$


The MCs for this spacetime for the covariant (or contravariant) stress-energy tensor form the following ten dimensional Lie algebra[19]

$$
\begin{aligned}
& \mathbf{X}_{1}=\partial_{x}, \\
& \mathbf{X}_{2}=\partial_{y}, \\
& \mathbf{X}_{3}=\partial_{z}, \\
& \mathbf{X}_{4}=z \partial_{x}-x \partial_{z}, \\
& \mathbf{X}_{5}=y \partial_{z}-z \partial_{y}, \\
& \mathbf{X}_{6}=x \partial_{y}-y \partial_{x}, \\
& \mathbf{X}_{7}=\partial_{t}, \\
& \mathbf{X}_{8}=t \partial_{x}-\epsilon x \partial_{t}, \\
& \mathbf{X}_{9}=t \partial_{y}-\epsilon y \partial_{t}, \\
& \mathbf{X}_{10}=t \partial_{z}-\epsilon z \partial_{t},
\end{aligned}
$$

where $\mathbf{X}_{7}, \ldots, \mathbf{X}_{10}$ are non-isometric MCs. In the above $\epsilon=+1$ or -1 , and $d t=$ $\sqrt{A / B} d \tilde{t}$. Further, we have assumed that $B$ is a constant, but even if we drop this assumption, we again get a ten dimensional algebra[19]. In contrast to this, if we use the mixed form of the stress-energy tensor, equations (6) do not constrain the vector sufficiently and we get the following form of the MC vector with infinite degrees of freedom

$$
\begin{aligned}
& \xi^{0}=0, \\
& \xi^{1}=f(x, y, z), \\
& \xi^{2}=g(x, y, z), \\
& \xi^{3}=h(x, y, z),
\end{aligned}
$$

where $d, f, g$ and $h$ are arbitrary functions. However, if the $T_{\nu}^{\mu}$ are independent of the time coordinate, we get $\xi^{0}=d(t), S$ becomes constant and spacetime turns out to be Minkowski (and we again have an infinite dimensional algebra).

Finally we remark that when the stress-energy tensor is written in the covariant form its symmetries can be compared with those of the Ricci tensor (the RCs) [14, 15]. (In this case it may be more appropriate to call them Einstein collineations as they are essentially collineations of the Einstein tensor, by the Einstein Field Equations.) For the contravariant form the number of independent equations being the same as in the covariant form, the number of symmetry generators would be the same. It is worth checking whether the collineations are directly related (through constants 
or at most the metric coefficients as linear combinations) or not. In principle the presence of the metric tensor could introduce major differences.

Further, the dimensionality of the Lie algebra of collineations also depends on the degeneracy of a tensor. The metric tensor is non-degenerate, therefore, its symmetries are always finite dimensional. When the stress-energy tensor is written in covariant form it always has a finite dimensional Lie algebra if it is non-degenerate (i.e. when $\left.\operatorname{det} T_{a b} \neq 0\right)$. As such, the results on MCs of the non-degenerate tensor in Ref. [24] (cases on pages 5151, 5152 and four cases in Table III) are obviously incorrect. Similarly, the infinite dimensional Lie algebras for the non-degenerate Ricci tensor claimed in Ref. [25] (Theorems 8, 9, 11 and 12, and the corresponding claim in the Abstract) also cannot be correct.

Notice that different valences of the stress-energy tensor correspond to different symmetry algebras. One needs to select the definition of matter collineations according to the physical application. For example, to construct conservation laws in the Hamiltonian formalism, symmetries of the contravariant tensor would be most appropriate.

It is worth mentioning that there is also an ambiguity in the definition of collineations for tensors of rank four. In particular, Weyl collineations[26] require more detailed analysis as the Weyl tensor could be defined as the trace-free part of the curvature tensor[2], in which case its valence would be $\left(\begin{array}{l}1 \\ 3\end{array}\right)$. Alternatively, as defined from spinor considerations[27] it should be regarded as covariant, i.e. of valence $\left(\begin{array}{l}0 \\ 4\end{array}\right)$. For 4 dimensions, the latter set is of 20 equations while the former is of $96-16-10=70$ equations (from the skew symmetry of the last pair of indices, the first Bianchi identities and the fact that the Weyl tensor is trace-free).

\section{Acknowledgments}

The authors are grateful to Ugur Camci for his help during the write-up of this paper. They also thank the 30th International Nathiagali Summer College on Physics and Contemporary Needs where this work was partly done. KS acknowledges a research grant from the Higher Education Commission of Pakistan.

\section{References}

[1] G. H. Katzin, J. Levine and W. R. Davis, J. Math. Phys. 10, 617 (1969).

[2] G. S. Hall, Symmetries and Curvature Structure in General Relativity (World Scientific Publishing Co., 2004). 
[3] K. L. Duggal and R. Sharma, Symmetries of Spacetimes and Riemannian Manifolds (Kluwer Academic Publishers, 1999).

[4] H. Stephani, D. Kramer, M. A. H. MacCallum, C. Hoenselaers and E. Herlt, Exact Solutions of Einstein's Field Equations (Cambridge University Press, 2003).

[5] J. Carot, J. da Costa and E. G. L. R. Vaz, J. Math. Phys. 35, 4832 (1994).

[6] G. S. Hall, I. Roy and E. G. L. R. Vaz, Gen. Rel. Grav. 28, 299 (1996).

[7] A. Qadir, K. Saifullah and M. Ziad, Gen. Rel. Grav. 35, 1927 (2003).

[8] W. R. Davis and G. H. Katzin, Am. J. Phys. 30, 750 (1962).

[9] W. R. Davis, L. H. Green and L. K. Norris, Nuovo Cimento B 34, 256 (1976).

[10] D. R. Oliver Jr and W. R. Davis, J. Math. Phys. 17, 1790 (1976).

[11] M. Tsamparlis and D. P. Mason, J. Math. Phys. 31, 1707 (1990).

[12] A. H. Bokhari and A. Qadir, J. Math. Phys. 34, 3543 (1987).

[13] A. H. Bokhari, M. Karim, D. N. Al-Sheikh and F. D. Zaman, Int. J. Theor. Phys. 47, 2672 (2008).

[14] K. Saifullah, Nuovo Cimento B 118, 1137 (2003) (Special issue devoted to Proc. "Black Holes, Gravitational Waves and Cosmology", Rome, Pescara, 2002).

[15] K. Saifullah, Int. J. Mod. Phys. D 14, 797 (2005).

[16] R. Maartens, S. D. Maharaj and B. O. J. Tupper, Class. Quantum Grav. 12, 2577 (1995).

[17] R. Maartens and S. D. Maharaj, Class. Quantum Grav. 3, 1005 (1995).

[18] A. di Teodoro and V. M. Villalba, Int. J. Theor. Phys. 47, 1885 (2008).

[19] U. Camci and A. Barnes, Class. Quantum Grav. 19, 393 (2002).

[20] U. Camci and M. Sharif, Class. Quantum Grav. 20, 2169 (2003).

[21] R. Penrose, The Road to Reality (Alfred A. Knopf, 2005).

[22] L. D. Landau and E. M. Lifshitz, The Classical Theory of Fields (Pergamon Press, 1975).

[23] C. W. Misner, K. S. Thorne and J. A. Wheeler, Gravitation (W. H. Freeman and Co., 1973). 
[24] M. Sharif, J. Math. Phys. 44, 5141 (2003).

[25] M. Ziad, Gen. Rel. Grav. 35, 915 (2003).

[26] I. Hussain, A. Qadir and K. Saifullah, Int. J. Mod. Phys. D 14, 1431 (2005).

[27] R. Penrose and W. Rindler, Spinors and Space-time (Cambridge University Press, 1986). 